\title{
Suggestions and tips regarding polymyxin B-immobilized fiber column direct hemoperfusion of neonates with sepsis
}

\author{
Naoto Nishizaki \\ Department of Pediatrics, Juntendo University Urayasu Hospital, Chiba, Japan
}

\section{Dear Editor:}

I read with great interest the case report entitled, "A successful application of adult polymyxin B-immobilized fiber column hemoperfusion to a neonate with septic shock" by Kim et al [1]. In this case, a female neonate was born at a gestational age of 38 weeks with a birth weight of $3.0 \mathrm{~kg}$. She developed late-onset septic shock caused by a carbapenem-resistant Acinetobacter baumannii infection and was treated with extracorporeal membrane oxygenation (ECMO) and adult polymyxin B-immobilized fiber column direct hemoperfusion (PMX-DHP). These interventions yielded significant short-term improvements in cardiopulmonary function. However, the patient died of multiorgan dysfunction syndrome two days after discontinuation of ECMO and PMX-DHP. The authors concluded that PMX-DHP could be used as an adjunctive treatment for selected neonates with Gram-negative bacterial septic shock. Although I partly agree with the authors, my colleagues and I recently reported that the effectiveness of PMX-DHP was limited in neonates with septic shock due to late-onset sepsis (onset $>72$ hours after birth) [2]. I wish to comment on the use of PMX-DHP for neonatal septic shock and provide suggestions based on our experiences.

At our institution, we identified 49 neonates with blood culture-proven sepsis among 1,115 neonates born between January 2013 and December 2018. Of these 49 neonates, six and five patients with septic shock had undergone PMX-DHP treatment for early-onset sepsis (EOS group) or late-onset sepsis (LOS group), respectively. We determined that the time from the decision to treat until treatment induction was significantly shorter in the EOS group than in the LOS group. The rates of in-hospital mortality and mortality after 28 days of treatment were significantly lower in the EOS group than in the LOS group. Interestingly, interleukin-6 (IL-6) level significantly decreased after treatment in the EOS group compared with the LOS group ( 8,396 vs. $1,235 \mathrm{pg} / \mathrm{ml}, \mathrm{P}=0.037)$. Our results have raised concerns regarding the following aspects. (1) Because EOS can be indicated earlier than LOS based on perinatal information (e.g., signs of maternal infection), PMX circuit can be promptly readied in such cases. (2) Vascular access is a prerequisite for prompt initiation of PMX-DHP, and this can be achieved relatively easily via umbilical vessel catheterization in EOS patients. (3) Although the original mechanism of action behind PMX-DHP therapy is direct adsorption of circulating endotoxin, the PMX-DHP column has also been reported to remove inflammatory cells. Thus, removal of activated inflammatory cells can have a tertiary effect of reducing the circu-

\section{Letter to the Editor}

Received: May 9, 2020

Revised: July 9, 2020

Accepted: July 9, 2020

Corresponding author Naoto Nishizaki Department of Pediatrics, Juntendo University Urayasu Hospital, 2-1-1 Tomioka, Urayasu, Chiba 279-0021, Japan

Tel: +81-47-53-111

Fax: +81-47-353-0526

E-mail: nishizak@juntendo.ac.jp

Copyright (@) 2020 The Korean Society of Critical Care Medicine

This is an Open Access article distributed under the terms of Creative Attributions Non-Commercial License (https:// creativecommons.org/li-censes/by-nc/4.0/) which permits unrestricted noncommercial use, distribution, and reproduction in any medium, provided the original work is properly cited. 
lating levels of inflammatory mediators, such as IL-6. Based on our results, we concluded that early reversal of vital signs of shock induced by PMX-DHP is more effective in EOS patients than in LOS patients. I note that Kim et al. [1] did not report the patient's IL-6 level as a measure of the severity of septic shock. IL-6 is considered useful as a marker of shock and a criterion for induction of PMX-DHP in sick neonates $[3,4]$.

Although a PMX-DHP column specialized for neonates with a priming volume of only $8 \mathrm{ml}$ (Toraymyxin 01-R; Toray Medical Co., Tokyo, Japan) is available in Japan, the effect of PMX-DHP in LOS may be limited, as reported by Kim et al. [1]. Furthermore, I believe that induction of PMX-DHP should be considered primarily for correcting hypercytokinemia instead of treating bacteria identified upon gram staining. Finally, I hope that PMX-DHP columns with low priming volumes (rather than adult PMX-DHP columns) can be applied to Korean neonates in the future and suggest that a further evaluation of neonatal sepsis is warranted.

\section{CONFLICT OF INTEREST}

No potential conflict of interest relevant to this article was reported.

\section{REFERENCES}

1. Kim YA, Kim H, Kim YM, Park SE. A successful application of adult polymyxin B-immobilized fiber column hemoperfusion to a neonate with septic shock. Acute Crit Care 2019;34:284-8.

2. Nishizaki N, Hara T, Obinata K, Nakagawa M, Shimizu T. Clinical Effects and outcomes after polymyxin B-immobilized fiber column direct hemoperfusion treatment for septic shock in preterm neonates. Pediatr Crit Care Med 2020;21:156-63.

3. Nishizaki N, Nakagawa M, Hara S, Oda H, Kantake M, Obinata K, et al. Effect of PMX-DHP for sepsis due to ESBL-producing E. coli in an extremely low-birthweight infant. Pediatr Int 2016;58:411-4.

4. Nishizaki N, Hirano D, Miyasho T, Obinata K, Shoji H, Shimizu T. Evaluation of urinary IL-6 in neonates with septic shock treated with polymyxin B-immobilized fiber column. Pediatr Int 2017;59:1032-3. 